\title{
Research of Ubiquitous Learning Cloud Service Platform
}

\author{
Weixing Xu \\ School of Energy and Power Engineering, \\ Jiangsu University \\ Zhenjiang, China \\ wilsonxujs@163.com
}

\author{
Mingyue Fan*, Dong Liu, Fei Tian \\ School of Management \\ Jiangsu University \\ Zhenjiang, China \\ *Corresponding author
}

\begin{abstract}
With the rapid development of modern science and information technology, the wide application of internet has offered a new carrier and channel for course teaching, while also brought many new challenges. The ubiquitous learning has become an important developing direction for future learning. Based on a new type ubiquitous learning and cloud architecture mode, this paper aimed at the research on the network space system in ubiquitous learning to produce innovative design for learning cloud space, which is a formal course in the cloud, seamless connection in the informal learning space, and the combination of subject learning space and three spatial blending learning styles. This learning style will be knowledge-based theoretical study, problem-oriented practical study and lifestylebased themes to learn seamless connection and bring about the interest-oriented generalization daily life. Through studying the design and architecture of space system module, it shows that this learning space can support the learning development of ubiquitous learning. And by using it, students are more interested and more active and teaching effect is very good.
\end{abstract}

Keywords-Ubiquitous learning; Learning space; Seamless learning; Cloud service platform

\section{INTRODUCTION}

With the rapid development of information technology, especially from the Internet to the mobile Internet has created a new way of life, work and learning across time and space, which has radically changed the methods of knowledge acquisition. The effective learning depends on the creation of a technology-supported, intelligent, seamless learning space. With the popularization of the intelligent terminal, Let the education resources restricted by the region accelerate to the direction of virtualization, openness, and sharing, such as direction, at the same time also makes the user at anytime, anywhere, on-demand access to services, teaching and learning cannot restricted by time, space and site conditions, knowledge acquisition channels of flexible and diverse, fragmentation, life-oriented learning anytime and anywhere will be possible, the concept of the ubiquitous learning has increasingly become a mainstream[1].

\section{UBIQUITOUS LEARNING}

With the popularization of wireless communication and the widespread use of mobile intelligent devices, the learning space of the learners has been greatly expanded, making it possible to learn anywhere and anytime. So, this new learning model has become a popular learning model. The ubiquitous learning refers to the process by anyone, at any time and any place, obtains any required learning resources based on any computer or mobile communication device and enjoys the ubiquitous learning service [2]. In the context of ubiquitous technology and pervasive computing, the learning content and cognitive goals, and actively using accessible learning resources to carry out learning activities. The characteristics of the general ubiquitous learning is Four-A (including Anyone, Anytime, Anywhere, Any device).There are many theoretical researches on the ubiquitous learning in China, but there are few researches on application such as system construction and network learning space. We innovatively expand Four-A to The new learning mode. The new model of learning including that anyone, anywhere, anytime, any device, anyway, any contests, and any learning support. The establishment of the The new model, in favour of improving the connotation of the learning, and letting the learners anywhere, anytime you can simply communicate to share, learning activities, theme discussion of generic network education space to provide theoretical support in their learning.

\section{RESEARCH OF UBIQUITOUS LEARNING CLOUd SERVICE PLATFORM}

The learning space is the basis of the all teaching activities. The network learning space is based on a certain learning support service platform, which provides private and personalized personal learning for each learner. Study space and public learning space for public service functions. Based on the cloud computing, the big data technology, and the analysis technology, the network learning space should have the four "elements" (character space, tools space, content space and process information space), the four "platform" (network platform, social platform, management platform and service support platform), and the five "target", namely the learners through the network space use a variety of cognitive tools for resources to carry out the study. At the same time with others, teachers or experts to develop social relation network, the establishment of close connection, in their 
interactions with promoting knowledge construction, improve the ability to solve the problem, and finally in the unceasing study development of individual and collective wisdom[3,4]. The platform is designed and implemented through three different types of learning.

\section{A. Formal Courses in the Cloud}

The formal course learning in the cloud refers to the formal learning of learning resources and the teachers' teaching based on the use of various curriculum resources in the network space. The system uses the knowledge points as the basic unit of the construction of learning space. According to the specification of curriculum and teaching, the curriculum is decomposed into the knowledge points one by one. The system for each knowledge point cloud, assigned to a study on the knowledge, storage teacher teaching video, a line of engineer technology practice video and interpretation of data. The simulation practice teaching resources, interactive learning comments and notes, and online measurement, etc. The learning cloud space corresponds to a knowledge point, and a series of knowledge points, namely the scientific combination of the learning cloud space, can form a professional course learning resource system [5].

\section{B. Seamless Informal Learning in the Cloud}

The informal learning of seamless connection refers to the learning process of fragmentation in human activities such as life, work, travel and socialization. The system uses the problem as the basic unit to construct the learning space. The system to provide the private study space of the cloud, and around the problem, store the learner through mobile phones and other portable devices photographs, video, sound recording the scene, written text, notes, etc. Whenever and wherever the records were exposed to the new things and the new problems, the new phenomena and the new knowledge.

\section{Thematic Learning in the Cloud}

Thematic learning refers to the phenomenon of social life or a particular aspect of learning content, such as a career or life need of the knowledge, skills, learning, some sports, literature and art, love of learning, etc. For thematic learning, it is generally initiated by one or more individuals with the common learning process [6]. The system with the topic as the basic unit for the construction of the learning space, and provide personal learning space friends sharing function and multiple users at the same time use a class of learning space function, these functions can make a people participate in the theme of the study, by sharing his life and interests, learning, feelings.

The three different types of learning is not unrelated three modules, learners, when they are in a study can be related to the knowledge, problems, topic three spatial unit in personalized free combination, through the study of the knowledge space solution theory knowledge. The problems learning space to solve practice operations. The model of flood in the study to theoretical study, practice learning, life application seamless integration, can let the user interests oriented, rather than the course oriented, accidental, individualized learning and interactive communication, provide convenience for our life and work.

\section{DESIGN AND ARCHITECTURE OF LEARNING CLOUD SERVICE PLATFORM}

\section{A. Design of Learning Cloud Service Platform}

Based on the new learning mode, as well as formal courses in the cloud, seamless informal learning, broad thematic learning three kind of study way, to design the function module of the learning loud space platform, as shown in figure 1 . 


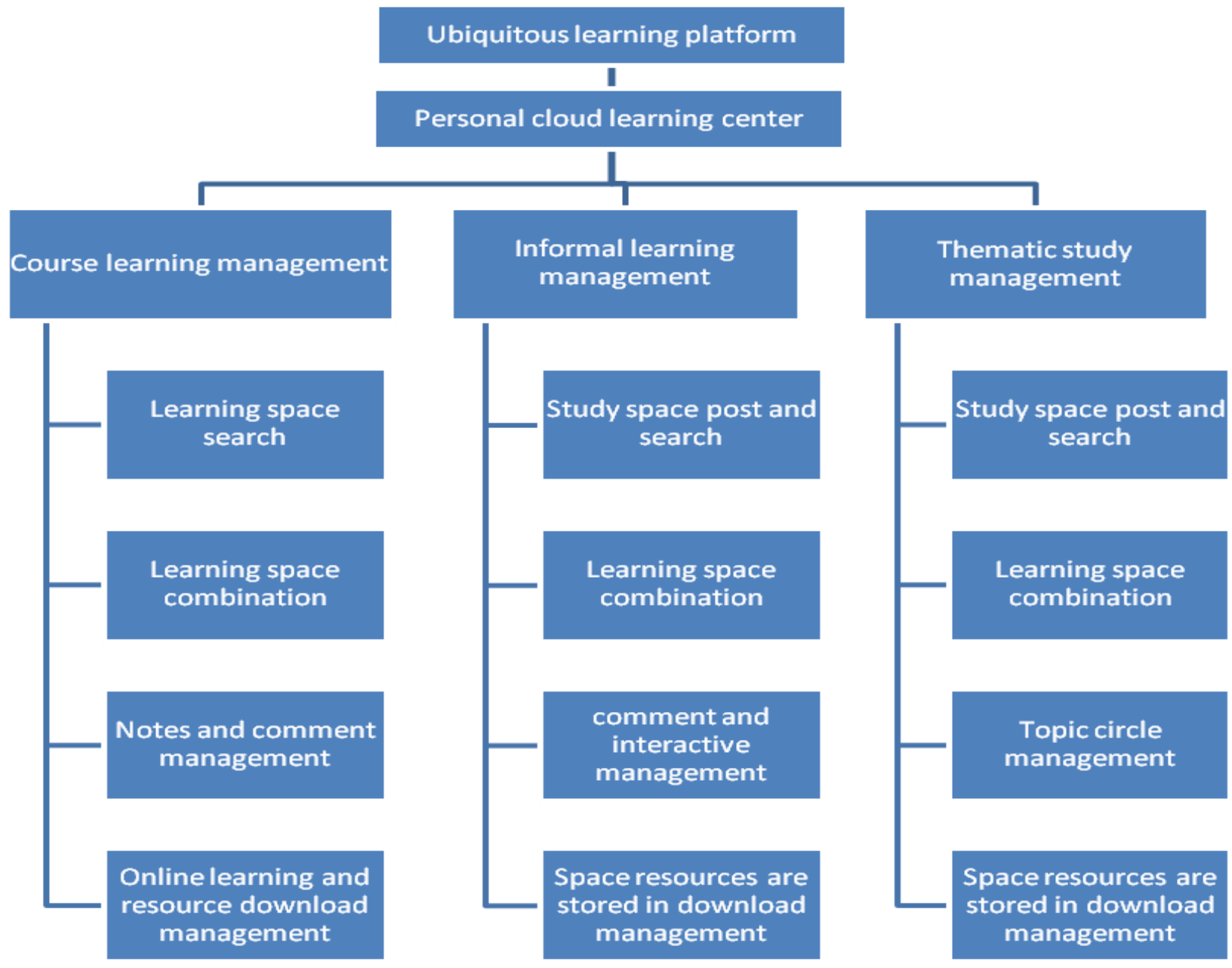

Fig. 1 Module of Learning Cloud Space Platform

Set up personal cloud learning center in the ubiquitous learning platform for system management, and course management, informal learning, thematic learning management three management module, respectively for knowledge learning space, the problem space, the topic learning space systems management. The knowledge learning space does not allow users to build or custom, only by the system according to the specific course outline or standard setting, the formation of the knowledge learning space group, and to study space for unit storage is associated with the knowledge of teacher video, technical documents, digital content such as teaching material. The user can search the learning space for the subject, and combine various knowledge points according to the requirements. In the personal cloud learning center, the user can establish his own combination for personalized learning [7]. During the course of study, users can make online notes and comments, etc., and download various teaching materials according to their permissions. The problem learning space allows the user to build or customize learning space, and according to the problems of its own use of portable devices anytime and anywhere to upload text, images, voice, video, and Internet access to relevant information and data. The problem learning space can set up private or public permission, and users can use the management module to retrieve the ask that other users share publicly. The problem learning space and individualized combination of the personal cloud learning center and other learning spaces, and the problem oriented fragmentation is learning. The topic learning space permissions more diversity, can be set to private space, open sharing of space, can also be visible to a specific group of friends, friends list and set up the space, form friends space, dynamic display update friends learning space resources. You can even set up multiple friends to share the same topic learning space. Users can access to retrieve friends the topic learning space resources, to establish the same topic space, and in the personal cloud learning center subject of all similar content learning space for the combination of personalized, can even and the knowledge, the problems learning space hybrid combination, formed its own unique learning circle, and friends the exchange of learning knowledge and asked, share the learning and experience, for seamless integration of ubiquitous learning.

\section{B. Architecture of Learning Cloud Service Platform}

The cloud architecture of learning cloud service flatform is divided into three layers, including: the software (SaaS), the platform (PaaS), and the infrastructure (IaaS) [8], as shown in figure 2 . 


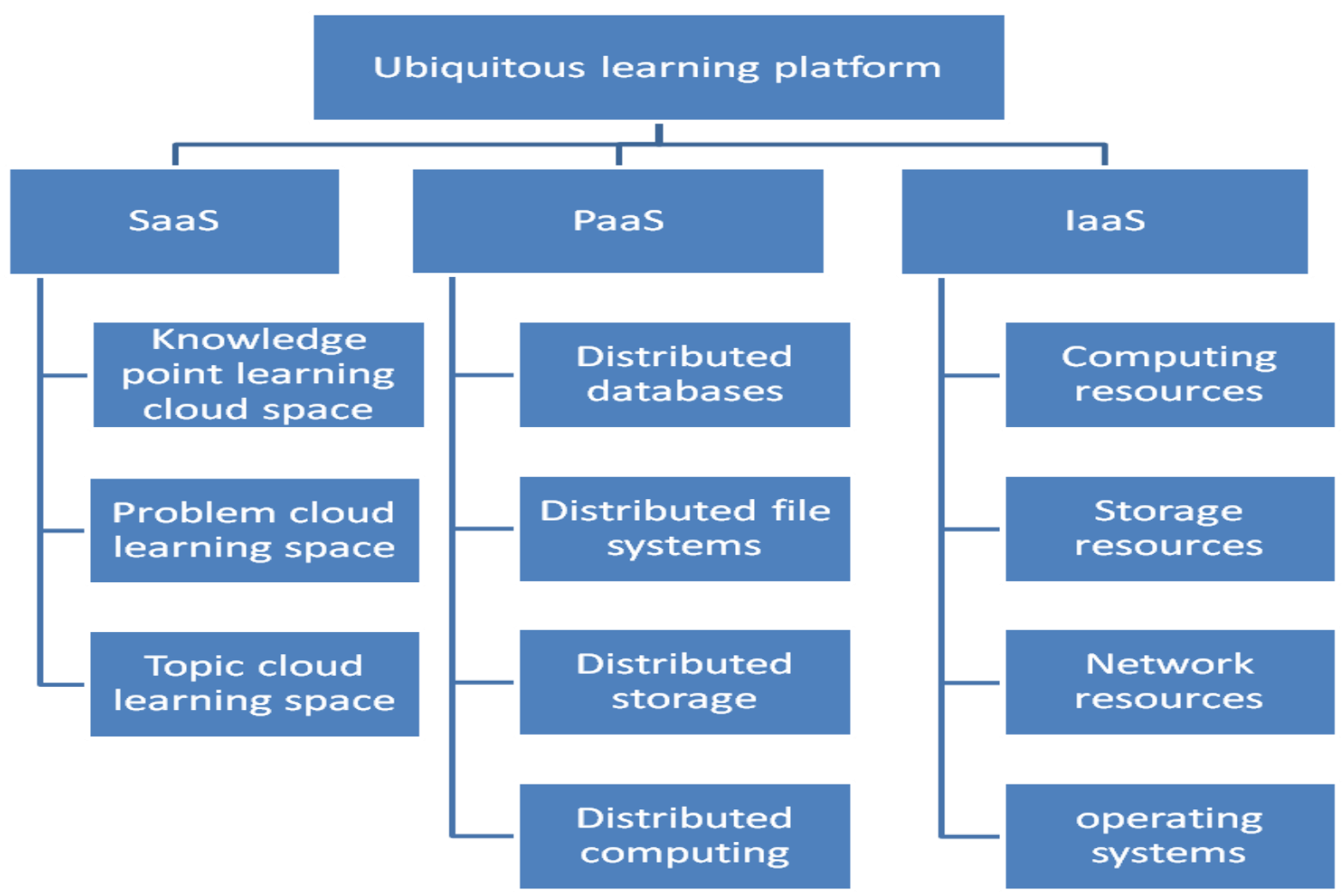

Fig. 2 Architecture of Learning Cloud Service Platform

The SaaS mainly uses the internet as the carrier, with the browser as the interactive mode. The server side of the program software to the remote users to provide the application model of software services in the ubiquitous learning network space. In the design, the SaaS package includes a person cloud learning center, the knowledge point learning cloud space, the problem cloud learning space, the topic cloud learning space. The learners can access and study the cloud space through virtual network access. When a large number of users view online frequently use and stay cloud, the front desk page to the user through virtual cache technology in real-time learning content, and the same file packet download which won't be interrupt for the network, and won't cause any changes to the cloud of metadata. When the user interacts or writes data in the cloud space, the data transfer to the cloud storage backend computer cluster will read and write to the metadata database without any impact on the foreground.

The PaaS facing the developers and administrators of the ubiquitous learning network space. The PaaS provides users with distributed databases, distributed file systems and distributed storage via the Internet. Distributed computing, action the basis of virtual storage, users can use the virtual operating system to develop and manage the capacity, function, permission and payload of learning cloud space. The retrieval and interaction of learning space are set up and managed.

The IaaS include computing resources, storage resources, network resources, operating systems, and middle. The cloud architecture includes cloud computing management nodes and cloud storage management nodes. When the learners use the learning space, the cloud storage management node distributes cloud storage space through the virtual operating system. The user uses the portable device to use multiple cloud space and the real-time interaction and storage of the cloud space. The cloud computing management node is responsible for the scheduling and storage management of all cloud storage Spaces. However, a large number of teaching and learning resources are stored in the cloud storage management node. Through the cloud computing management node, efficient data management is conducted and the ordered feedback is given to the users.

\section{APPLICATION OF LEARNING ClOUd SERVICE PLATFORM}

Based on the ubiquitous learning space design model and principles, the School of Energy and Power Engineering of Jiangsu University established the open teaching digital laboratory of the energy and power control system, as shown in figure 3. The application of a compatible multi-screen responsive framework enables learners to take advantage of the learning equipment they can acquire and acquire the information that they need in their own way [9]. The registered login can create learning cloud space, users can use their own independent learning space to create online classes. The online learning is available on all kinds of mobile terminals. We should learn from the knowledge of life without limitation, and learn with the knowledge of life. 


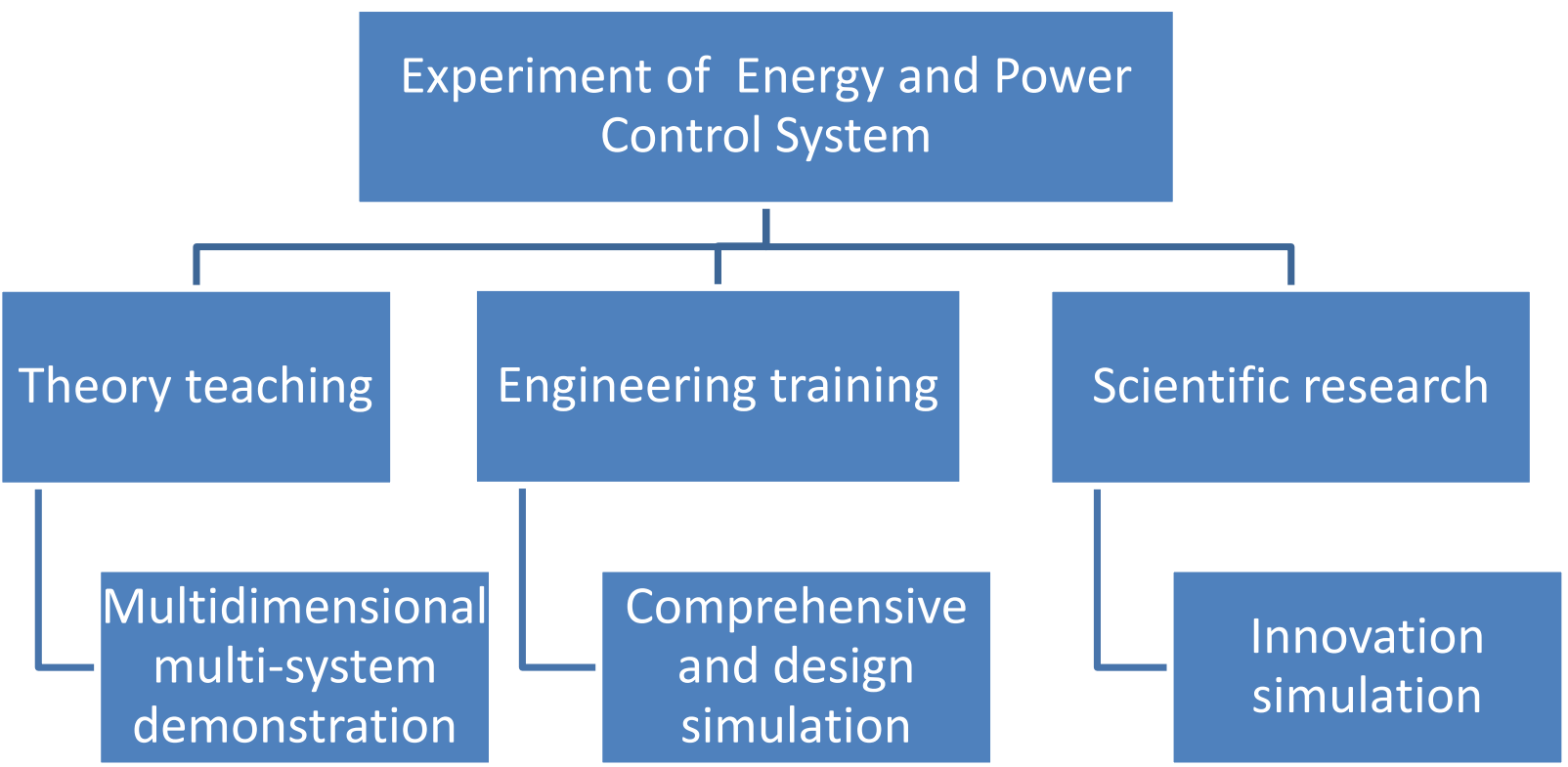

Fig. 3 Application of Energy and Power Engineering Control System

\section{CONCLUSION}

In this paper, the new type learning mode of the ubiquitous learning online space was designed and researched. Three learning clouds were formally put forward. Including the formal courses space, the seamless informal learning space, the thematic learning space, and the three hybrid combination way of learning. The learning method combines theory learning, practical learning, and life application learning seamlessly, and realizes the ubiquitous learning with the interest oriented universalization and daily life. Through studying the design and architecture of space system module, it shows that this learning space can support the learning development for ubiquitous learning. And by using it, students are more interested and more active. The teaching effect is very good.

\section{ACKNOWLEDGMENT}

This research was financially supported by the Top-notch Academic Programs Project of Jiangsu Higher Education Institutions (PPZY2015A029), the Priority Academic Program Development of Jiangsu Higher Education Institutions (PAPD) , Postdoctoral Research Project of Suzhou Sinye Materials technology Co., Ltd., the Development and Demonstration Project of Jiangsu Province (BE2016341), the Teaching Reform Project of Jiangsu University(2017JGYB008), the Teaching Reform Project of Jiangsu University Jingjiang college(13986jg2017B020), and the Teaching Reform Project of Committee for Education(NDXGK2017Y-03), Postgraduate Education Reform Project of Jiangsu Province(JGLX_083), Scientific Research Foundation of Jiangsu University (14JDG178).

\section{REFERENCES}

[1] Zhao Huiqin, Zhang Tianyun, Wang Li, Constucting a Context-aware Computing Personal Learning Space in Ubiquitous Learning Environment, China Educational Technology,354(2016) 29-35.

[2] Wu Di, Peng Xian, Zhang Jiaqiong, Research on the Standard System of Cloud Based Education Service, 21(2015)92-100.

[3] Nie Xuejun, Du Minjie, Development of Virtual Experiment Platform Based on Mechanical Engineering Control, China Modern Educational Equipment,15(2016)4-6.

[4] Zhu Tangchun, He Qiangqiang, Meng Lili, Zhang Weiqi, Design of Control Diagram Virtual Experiment Platform, Mechanical Engineer, 12(2015)150-151.

[5] Wu Zheng, Hao Wenqing, Research on the Network Learing Space for Everyone System Based on Cloud Architecture Model, China Educational Technology,350(2016) 65-68.

[6] Pang Xinyu, Lian Zisheng, Zhang Linyan, The Virtual Experimental Technology in"Mechanical Control Virtual Experimental System, Journal of Shanxi Datong University(Natural Science Edition),31(2015)69-72.

[7] Wang Wenfeng, Wang Jikai, Di Liming, Ding Weichao, 3D Virtual Experiment System for the Vehicle Velocity Control, Computer Simulation, 5(2015)182-185.

[8] Li Zhen, Zhou dongdai, Zhong shaochun, Research on Personalized Web Learning Space Based on Cloud Computing, 26(2016)114-120.

[9] Wang Wenfeng, Wang Jikai, Di Liming, Ding Weichao, "3D Virtual Experiment System for the Vehicle Velocity Control", China, Computer Simulation, 5(2015)182-185. 